\title{
A!
}

This is an electronic reprint of the original article.

This reprint may differ from the original in pagination and typographic detail.

Laitinen, Tiera; Jarvinen, Riku; Kallio, Esa; Janhunen, Pekka

\section{Forcing continuous reconnection in hybrid simulations}

Published in:

Physics of Plasmas

DOI:

$10.1063 / 1.4890854$

Published: 01/01/2014

Document Version

Publisher's PDF, also known as Version of record

Please cite the original version:

Laitinen, T., Jarvinen, R., Kallio, E., \& Janhunen, P. (2014). Forcing continuous reconnection in hybrid simulations. Physics of Plasmas, 21(7), 1-9. [072906]. https://doi.org/10.1063/1.4890854

This material is protected by copyright and other intellectual property rights, and duplication or sale of all or part of any of the repository collections is not permitted, except that material may be duplicated by you for your research use or educational purposes in electronic or print form. You must obtain permission for any other use. Electronic or print copies may not be offered, whether for sale or otherwise to anyone who is not an authorised user. 


\section{Forcing continuous reconnection in hybrid simulations}

T. V. Laitinen, R. Jarvinen, E. Kallio, and P. Janhunen

Citation: Physics of Plasmas 21, 072906 (2014); doi: 10.1063/1.4890854

View online: https://doi.org/10.1063/1.4890854

View Table of Contents: http://aip.scitation.org/toc/php/21/7

Published by the American Institute of Physics

\section{Articles you may be interested in}

Current disruption and its spreading in collisionless magnetic reconnection

Physics of Plasmas 20, 112101 (2013); 10.1063/1.4827828

\section{PHYSICS TODAY}

MANAGER'S GUIDE

WHITEPAPERS

\section{READ NOW}

PRESENTED BY Multiphysics Simulation 


\title{
Forcing continuous reconnection in hybrid simulations
}

\author{
T. V. Laitinen, ${ }^{1, a)}$ R. Jarvinen, ${ }^{1,2}$ E. Kallio, ${ }^{3,1}$ and P. Janhunen ${ }^{1}$ \\ ${ }^{1}$ Finnish Meteorological Institute, PL 503, FI-00101 Helsinki, Finland \\ ${ }^{2}$ Laboratory for Atmospheric and Space Physics, University of Colorado Boulder, 3665 Discovery Drive, \\ Boulder, Colorado 80303, USA \\ ${ }^{3}$ School of Electrical Engineering, Aalto University, PL 13000, FI-00076 Aalto, Espoo, Finland
}

(Received 13 March 2014; accepted 10 July 2014; published online 22 July 2014)

\begin{abstract}
We have performed hybrid simulations of driven continuous reconnection with open boundary conditions. Reconnection is started by a collision of two subsonic plasma fronts with opposite magnetic fields, without any specified magnetic field configuration as initial condition. Due to continued forced plasma inflow, a current sheet co-located with a dense and hot plasma sheet develops. The translational symmetry of the current sheet is broken by applying a spatial gradient in the inflow speed. We compare runs with and without localized resistivity: reconnection is initiated in both cases, but localized resistivity stabilizes it and enhances its efficiency. The outflow speed reaches about half of Alfvén speed. We quantify the conversion of magnetic energy to kinetic energy of protons and to Joule heating and show that with localized resistivity, kinetic energy of protons is increased on average five-fold in the reconnection in our simulation case. (C) 2014 AIP Publishing LLC. [http://dx.doi.org/10.1063/1.4890854]
\end{abstract}

\section{INTRODUCTION}

Reconnection is an integral part of the dynamics of many space plasma systems such as magnetospheres. Global simulations, most often based on magnetohydrodynamic (MHD) or hybrid description of plasma, are now a common tool for the study of such space plasma environments. To be able to model magnetospheric dynamics correctly, such simulations must also include reconnection, and they do: there are numerous publications on how reconnection has been identified in global MHD simulations, e.g., in the magnetotail $^{1,2}$ and on the magnetopause. ${ }^{3,4}$ Hybrid simulations are usually limited to a part of the magnetosphere in the Earth's case, but they too have been used to study reconnectionrelated phenomena, ${ }^{5,6}$ as well as limited MHD models. ${ }^{7}$ In all these simulations interest is usually focused on the wider context: conditions that cause reconnection, on its location(s), and on its consequences in the wider plasma system.

Detailed simulation studies of reconnection most often employ a quite different setup. A good example of "standard" initial and boundary conditions for reconnection simulations is the famous Geospace Environmental Modeling (GEM) reconnection challenge. ${ }^{8}$ The initial condition was a stationary Harris current sheet, with a small perturbation to create a seed $x$-line in the middle of the simulation box. Boundary conditions were periodic. When boundaries are periodic or closed, the system develops from the initial state through reconnection to a minimum magnetic energy configuration and stops there. This means that reconnection is necessarily of short duration. Such simulations are excellent for studying the spontaneous onset of reconnection, structure of the reconnection region, and for comparing the reconnection rate in different solvers, as was done in the GEM challenge. In contrast, they are not suitable for gaining insight into the long-

\footnotetext{
a) tiera.laitinen@fmi.fi
}

term stability of reconnection process, nor for studying the effect of fluctuations in the inflows. Also the outflow jets did not have enough space to develop fully and thereby could not be analyzed in the GEM challenge. With periodic or closed boundary conditions, one would need a huge simulation domain to allow for a realistic outflow process from the reconnection region.

Open boundary conditions have also been used in several earlier works. Here, an important further distinction is whether reconnection is driven or undriven. By undriven, we mean such boundary conditions at the inflow boundary that new plasma and magnetic flux can enter the simulation domain, but the rate at which this happens is not pre-specified. The inflow rate is thus determined only by the internal dynamics of the simulation. Undriven boundary conditions are not straightforward to implement. Nevertheless, they have been used in MHD, ${ }^{9}$ Hall MHD,${ }^{10}$ hybrid, ${ }^{11}$ and particle-in-cell ${ }^{12}$ simulations. In magnetospheric context, this kind of boundary conditions could be relevant to magnetotail reconnection.

The other alternative is to externally force, or drive, reconnection process in the simulation by specifying the inflow electric field ${ }^{13,14}$ or plasma velocity at the inflow boundaries. In this paper, it will be done by specifying the velocity distribution of new particles injected into the simulation domain at the inflow boundaries. Such boundary condition resembles the situation at the subsolar magnetopause, where the magnetosheath flow against the magnetopause provides the external forcing. ${ }^{15}$ Forced reconnection has also been modelled in a time-limited manner, e.g., in the Newton reconnection challenge. ${ }^{16}$ The subsolar magnetopause has been our primary motivation. In this paper, however, we settle for a symmetric configuration as that is the easiest way to obtain dynamic pressure balance of the inflows, which is necessary to make the reconnecting current sheet stay within the simulation domain.

We use a hybrid simulation code, which has previously been used for global simulations of solar wind and plasma 
interactions of several solar system objects. We use open boundary conditions with forced inflow to create continuous driven reconnection. We do not set up any current sheet or xline as an initial condition. Instead, we let the current sheet and $\mathrm{x}$-line form during the simulation as a result of the colliding inflow fronts. This setup is made to resemble reconnection regions that appear in global simulations as much as feasible, and we use it to study the ignition and appearance of reconnection in more detail than what is possible in real global simulations. We also study the effect of localized resistivity on the stability and rate of reconnection.

\section{SIMULATION SETUP}

\section{A. The HYB code}

The model used in this study, HYB-Reconnection, is based on the HYB hybrid simulation platform developed at the Finnish Meteorological Institute. The HYB code was first used to study solar wind interaction with Mars. ${ }^{17}$ It has then been applied to many other solar system bodies including Venus, ${ }^{18}$ Mercury, ${ }^{19}$ and the Moon, ${ }^{20}$ and recently also to exoplanetary environments such as a "magnetized Mars." 21 A brief summary including the physical equations solved by the model is given below (for details see Ref. 22). The numerical implementation is described extensively by Sillanpää. ${ }^{23}$

In the HYB model, ions are modelled as particles moving under the Lorentz force and electrons are a chargeneutralizing, massless fluid. Magnetic field is propagated on faces of cubic grid cells using Faraday's law. This construction ensures divergence-free magnetic field. The physical equations solved in the model are summarized as follows:

$$
\begin{gathered}
m_{i} \frac{d \overrightarrow{v_{i}}}{d t}=q_{i}\left(\vec{E}+\overrightarrow{v_{i}} \times \vec{B}\right) \\
\frac{d \vec{x}_{i}}{d t}=\vec{v}_{i} \\
\vec{E}=-\vec{U}_{e} \times \vec{B}+\eta \vec{J} \\
\vec{U}_{e}=\vec{U}_{i}-\frac{\vec{J}}{e n} \\
n_{i}=n_{e} \\
\frac{\partial \vec{B}}{\partial t}=-\nabla \times \vec{E} \\
\vec{J}=\frac{\nabla \times \vec{B}}{\mu_{0}},
\end{gathered}
$$

where $\mathrm{m}_{\mathrm{i}}, \mathrm{q}_{\mathrm{i}}, \mathrm{v}_{\mathrm{i}}$, and $\mathrm{x}_{\mathrm{i}}$ are the mass, charge, velocity, and position of a simulation ion. $\mathrm{E}$ and $\mathrm{B}$ are electric and magnetic field and $\mathrm{J}$ is the current density. $\mathrm{U}_{\mathrm{e}}$ and $\mathrm{U}_{\mathrm{i}}$ are the electron and ion bulk velocities and $n_{e}$ and $n_{i}$ are the electron and ion densities. $\eta$ is the pre-defined resistivity profile used in Faraday's law to introduce explicit magnetic field diffusion. $\mathrm{e}$ is the positive elementary charge and $\mu_{0}$ is the vacuum permeability.

Starting with positions and velocities of ions and magnetic field in the simulation domain, these equations give the time evolution of the system. Thus, ion dynamics are selfconsistently coupled with the electric and magnetic field.

\section{B. Units and normalisation}

As the HYB code was originally developed for planetary simulations, it was built to work in SI units. Therefore, the simulation parameters are presented in SI units also in this paper. Although this is unconventional in the field of "reconnection in a box" simulations, our choice of units has the advantage that it makes it easy to compare the results with observations and global simulations. To facilitate comparison with other simulation studies and theoretical considerations, where scaled units are preferred, we also provide some scaled values.

Scaling of units is somewhat arbitrary. The initial state is not a meaningful scaling reference, since the simulation is not initialized in a current sheet configuration. To calculate the scaling parameters, we have chosen to use numbers that approximately describe the plasma properties in the simulation results after the current sheet and reconnection outflows have fully formed. We take the values from the inflow region outside the current sheet, at $\mathrm{x}=-1000 \mathrm{~km}, \mathrm{y}=\mathrm{z}=0$ (the dot marked as "inflow timeseries measured here" in Fig. 1). After simulation initialization effects have vanished, plasma parameters have approximately constant values spatially from this point to the inflow boundary, and in the run with localized resistivity also temporally, as will be shown in Sec. III.

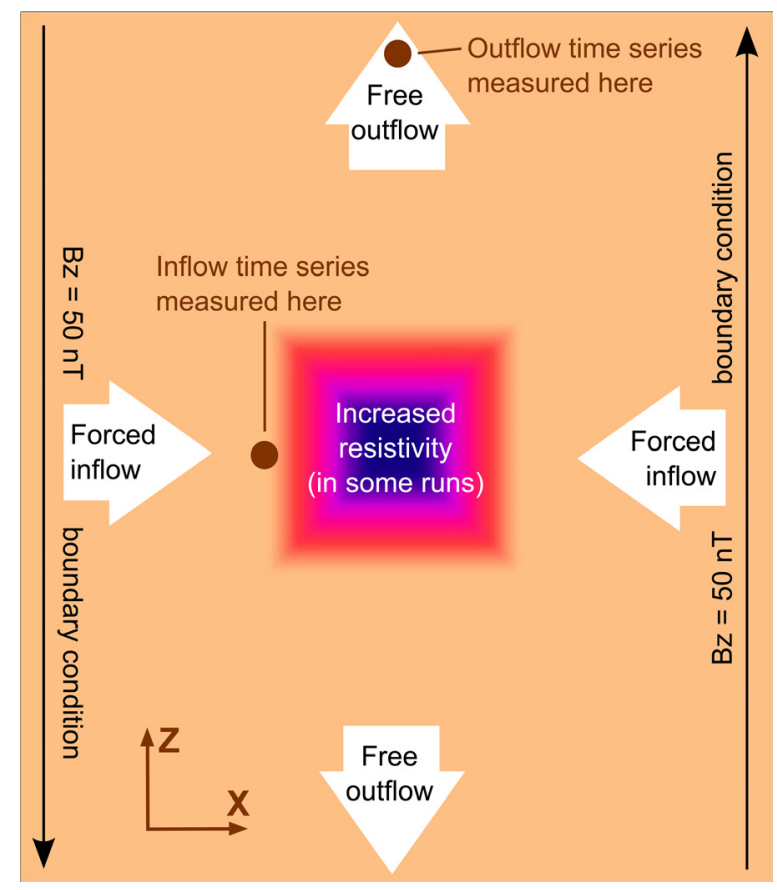

FIG. 1. The simulation setup. The simulation domain is in the $\mathrm{xz}$ plane, extending to $\pm 3000 \mathrm{~km}\left( \pm 43 \mathrm{c} / \omega_{\mathrm{pi}}\right)$ in the $\mathrm{x}$ direction and $\pm 3500 \mathrm{~km}$ $\left( \pm 50 \mathrm{c} / \omega_{\mathrm{pi}}\right)$ in the $\mathrm{z}$ direction. Background color shows the resistivity in Lrrun, ranging from $1.22 \eta_{0}$ to $3.67 \eta_{0}$. Particles are injected to the box at the walls perpendicular to the $\mathrm{x}$ direction with a thermal velocity distribution having a bulk velocity of $100 \mathrm{~km} / \mathrm{s}$ along the $\pm x$ axis. A \pm z-directed magnetic field is also imposed on the inflow walls. Neumann-type boundary conditions with free outflow of particles are applied in the $\mathrm{z}$ direction, and periodic boundary conditions in the y direction. The time series in Fig. 6 are measured at the brown dots: $\mathrm{x}=-1000 \mathrm{~km}, \mathrm{y}=\mathrm{z}=0$ for inflow and $\mathrm{z}=3000 \mathrm{~km}, \mathrm{x}=\mathrm{y}=0$ for outflow. 
The inflowing plasma is approximately characterized by the following numbers, which we choose as the basis of dimensionless units:

- Ion inertial length $\mathrm{c} / \omega_{\mathrm{pi}}=70 \mathrm{~km}=\lambda_{0}$

- Inverse of ion Larmor angular frequency $\omega_{\mathrm{ci}}{ }^{-1}=0.2$ $\mathrm{s}=\tau_{0}$.

From these follows:

- Nominal density $\mathrm{n}_{0}=10.6$ protons $/ \mathrm{cm}^{3}$

- Nominal magnetic flux density $\mathrm{B}_{0}=52.2 \mathrm{nT}$

- Nominal Alfvén speed $\mathrm{v}_{\mathrm{A} 0}=350 \mathrm{~km} / \mathrm{s}$ (neglecting electron mass)

- Nominal resistivity $\eta_{0}=30787 \Omega \mathrm{m}$.

\section{Boundary and initial conditions}

The simulation setup is illustrated in Figure 1. The dimensions of the simulation box are 120 cells in $\mathrm{x}$ direction, 20 cells in $\mathrm{y}$ and 140 cells in $\mathrm{z}$, each cell measuring $50 \mathrm{~km}$ $\left(0.71 \mathrm{c} / \omega_{\mathrm{pi}}\right)$ across. The origin is at the centre of the box and Figure 1, as all subsequent images, shows the $\mathrm{xz}$ plane at $\mathrm{y}=0$. Thus the extent of the simulation domain is $\pm 3000 \mathrm{~km}$ $\left(43 \mathrm{c} / \omega_{\mathrm{pi}}\right)$ in the inflow direction and $\pm 3500 \mathrm{~km}\left(50 \mathrm{c} / \omega_{\mathrm{pi}}\right)$ in the outflow direction.

At the beginning, the simulation box is filled with thin stationary plasma with no magnetic field. At $\mathrm{t}=0$ inflows are initiated. On the walls perpendicular to the $\mathrm{x}$ axis, particles are injected into the box following a thermal velocity distribution with a bulk flow speed of $100 \mathrm{~km} / \mathrm{s}\left(0.29 \mathrm{v}_{\mathrm{A} 0}\right)$ along the $\pm x$ axis. Towards the upper and lower edges of the box, the inflow speed decreases linearly to $80 \mathrm{~km} / \mathrm{s}$. (We have also performed test runs where this inflow velocity gradient is different or absent.) In addition, a $50 \mathrm{nT}\left(0.96 \mathrm{~B}_{0}\right)$ magnetic field is imposed at the inflow walls, directed along $\pm \mathrm{z}$ axis.

In the $y$ direction, periodic boundary conditions are applied. In the $\mathrm{z}$ direction, Neumann boundary condition is applied to the fields and particles are allowed to exit the simulation freely.

\section{Resistivity}

A grid-based simulation has two types of diffusion. Numerical diffusion is due to the discretization of the physical equations, while explicit diffusion is caused by diffusion terms in the equations. In the case of magnetic field, the amount of explicit diffusion in the model is controlled by a resistivity coefficient. We discuss only explicit resistivity here, but from the fact that even modest changes of resistivity have a notable effect on the simulation outcome, we deduce that numerical diffusion in the code is smaller than explicit diffusion in these runs.

Certain amount of explicit resistivity is necessary to keep the simulation runs stable. We use a background resistivity of a) $10 \mathrm{~s}$

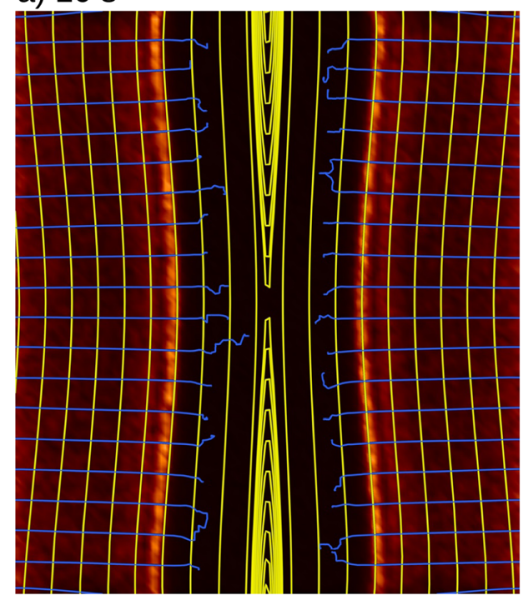

c) $20 \mathrm{~s}$

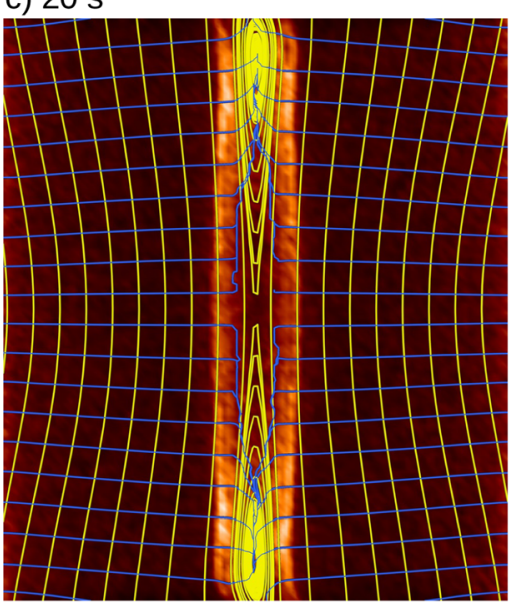

b) $15 \mathrm{~s}$

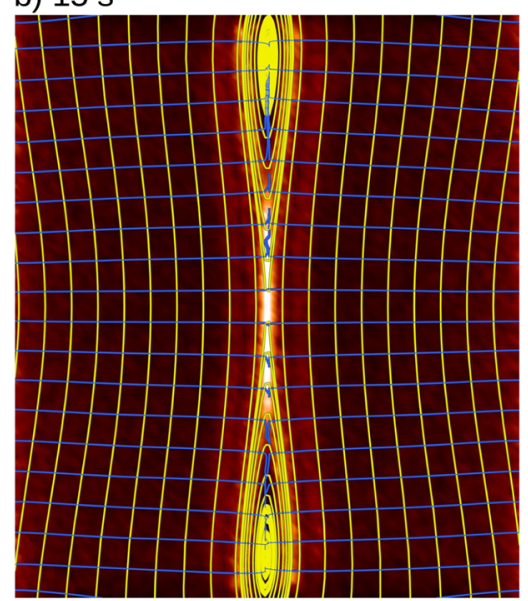

d) $40 \mathrm{~s}$

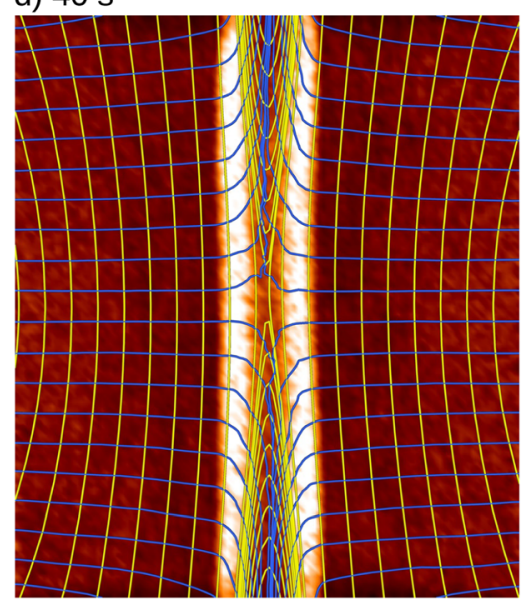

$\mathrm{n}\left[\mathrm{cm}^{-3}\right]$

$-30$

$-25$

20

FIG. 2. A cut in xz plane through the entire simulation domain at four selected times during the initialization of the simulation in the Cr-run. Magnetic field lines in yellow, plasma flow lines in blue, and plasma density $\left[\mathrm{m}^{-3}\right]$ represented by the background color. 

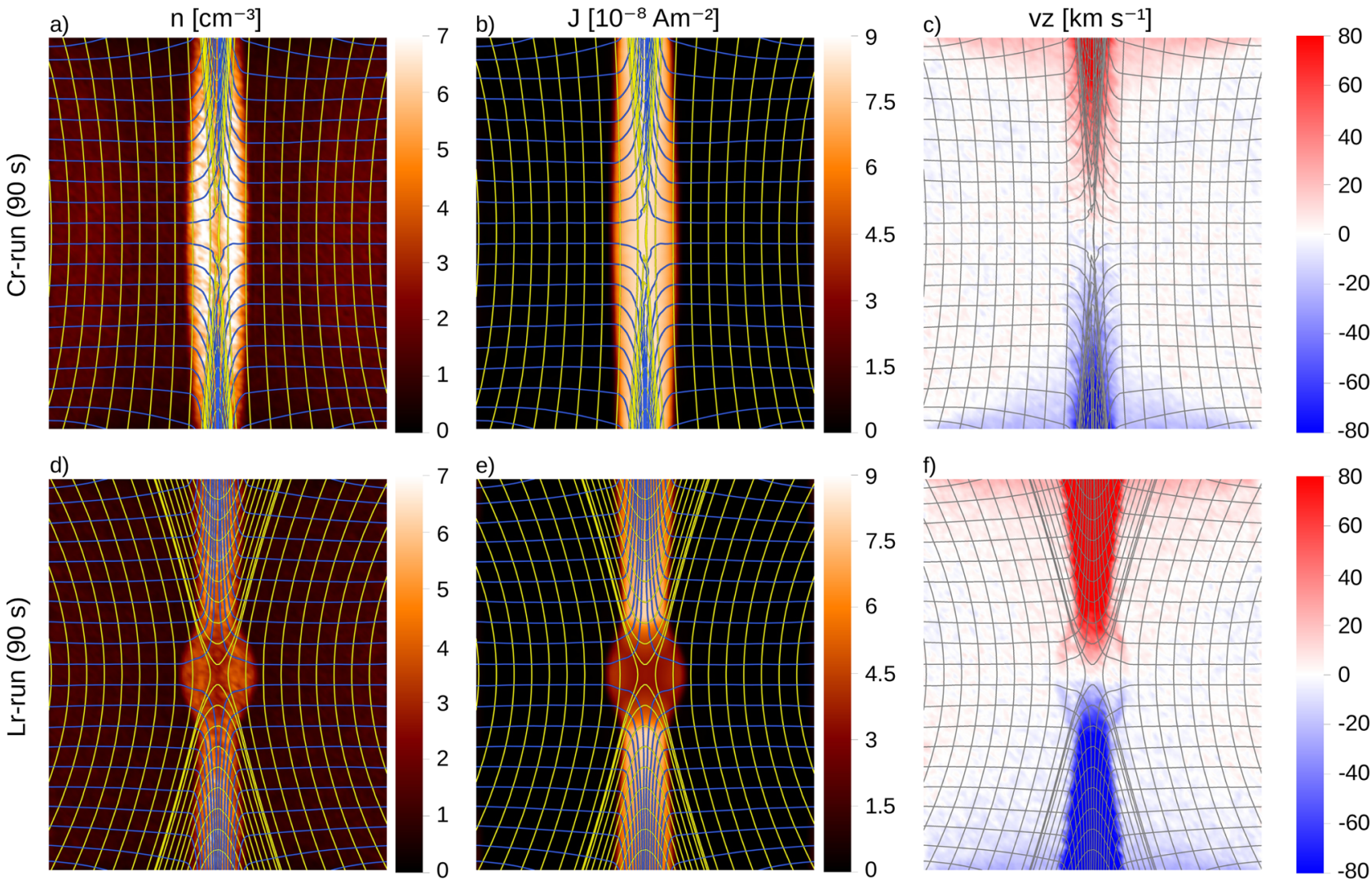

FIG. 3. Fully developed reconnection in its steady state $90 \mathrm{~s}\left(450 \omega_{\mathrm{ci}}{ }^{-1}\right)$ after the start of the simulation, a-c: Cr-run and d-f: Lr-run. Magnetic field lines in yellow, plasma flow lines in blue, except in $\mathbf{c}, \mathbf{f}$, where both in gray. Colour scale: $\mathbf{a}, \mathbf{d}$ : plasma density $\left[\mathrm{m}^{-3}\right]$, b, e: current density $\left[10^{-8} \mathrm{~A} / \mathrm{m}^{2}\right], \mathbf{c}, \mathbf{f}:$ outflow speed from the reconnection site $\left(\mathrm{v}_{\mathrm{z}}\right)[\mathrm{km} / \mathrm{s}]$ (note that the color scale in $\mathbf{f}$ is saturated, higher speeds are reached in Lr-run; see text).

$\eta_{\mathrm{bg}}=37700 \Omega \mathrm{m}\left(1.22 \eta_{0}\right)$. In this paper, we analyze in detail two runs, one of which has a constant resistivity (the background value) and one which is exactly similar in all other respects, but has a region of increased resistivity at the center of the domain. The resistivity increase is set up as follows. Within $|\mathrm{x}|$ and $|\mathrm{z}|<200 \mathrm{~km}\left(2.86 \mathrm{c} / \omega_{\mathrm{pi}}\right)$, resistivity is $\eta_{\mathrm{c}}=3$ $\eta_{\mathrm{bg}}=3.67 \eta_{\mathrm{o}}$. Outside $|\mathrm{x}|$ or $|\mathrm{z}|>1000 \mathrm{~km}\left(14.3 \mathrm{c} / \omega_{\mathrm{pi}}\right)$, resistivity has the background value of $\eta_{\mathrm{bg}}$. Between these limits resistivity changes linearly, as illustrated by the background colour in Figure 1. Henceforth, these two runs will be referred to as Cr-run (constant resistivity run) and Lr-run (localized resistivity run).

\section{RESULTS}

\section{A. Ignition}

Figure 2 illustrates the ignition of reconnection in the Cr-run. The magnetic geometry is shown by yellow field lines and plasma flow from the sides by light-blue flow lines. The flow lines are integrated in three dimensions, and if one ends inside the boundaries, it means that the flow line actually exited the simulation box through the y boundary. (Periodic boundary condition in y direction returns the flow to the box at the opposite boundary, but the flow line is no more drawn there.) Plasma density is shown by the color scale, which differs from one panel to another, because density increases strongly in the box during the initial phase of the simulation.
The first panel is $10 \mathrm{~s}\left(50 \omega_{\mathrm{ci}}{ }^{-1}\right)$ after simulation start. The inflowing plasma has formed two compression fronts that are about to collide. Field lines bend inward in the middle due to the imposed inflow velocity gradient. However, it can be seen that the magnetic field also tends to advance faster at the edges of the domain. This is at least partly because particles of the initial plasma escape from the simulation near the boundary, which leads to decreased pressure, so that the initial plasma opposes the inflow fronts less near the boundaries than elsewhere.

At $15 \mathrm{~s}\left(75 \omega_{\mathrm{ci}}{ }^{-1}\right)$, the plasma fronts have collided in the middle. Magnetic field lines have started to merge in the middle and at the edges.

At $20 \mathrm{~s}\left(100 \omega_{\mathrm{ci}}{ }^{-1}\right)$, an $\mathrm{x}$-point can be seen in the middle, and two plasmoids whose centres are near the boundaries. Plasma acceleration away from the X-point has started, but the outflows have not yet reached the boundaries. Plasma is thus accreting in the plasmoids and pushing them away from the x-point.

After $40 \mathrm{~s}\left(200 \omega_{\mathrm{ci}}{ }^{-1}\right)$, the outflows from the $\mathrm{x}$-point have reached their full speed even at the boundaries. The plasmoids have been blown out of the box.

\section{B. Resistivity effect on configuration}

The initial phase of the ignition is similar in both runs, with or without localized resistivity. However, after the first collision of incoming plasma fronts, the development is 
faster in Lr-run, and also morphological differences begin to arise. They are illustrated in Fig. 3, showing the configuration at $90 \mathrm{~s}\left(450 \omega_{\mathrm{ci}}{ }^{-1}\right)$ after simulation start, when reconnection has attained its maximum efficiency in both runs.

As one would expect, the localized resistivity increase enhances destruction of magnetic flux in the center of the domain in Lr-run, which causes the magnetic field lines to be more curved. Localized resistivity also causes widening of the current sheet and reduction of current density at the center (Fig. 3(e)). This is reflected also in the plasma density ( $3 \mathrm{~d})$. Comparing a to $d$ and $b$ to e in Fig. 3, one sees that the current sheet is approximately equally thick in both runs, but both current and plasma densities are much higher in $\mathrm{Cr}$-run in the entire current sheet, also outside the region where the value of resistivity is different in these two runs. This is because reconnection operates less efficiently in $\mathrm{Cr}$-run and both plasma and magnetic flux build up due to constant forced inflow combined with slower outflow than in Lr-run. The larger current density can also be understood from the point of view that when resistivity is lower, steeper magnetic gradient is needed to allow same rate of magnetic diffusion.

Comparing Figs. 3(c) and 3(f) shows that plasma acceleration away from the $\mathrm{x}$-point is more efficient and takes place on a more compact length scale in Lr-run. This can be interpreted as a consequence of the wider opening angle of the outflow cones, resulting from the stronger bend in inflowing magnetic fields: it implies larger normal component of the magnetic field in a thicker layer within the current sheet, which results in a larger $\vec{J} \times \vec{B}$ force.

Figure 4 shows density, temperature, current density, antiparallel magnetic flux density $B_{z}$, and ion inflow speed $v_{x}$ measured along the central normal of the current sheet ( $\mathrm{x}$ axis). Thin blue line represents $\mathrm{Cr}$-run and thick red line Lrrun. The profiles are qualitatively similar in both runs, although resistivity increase allows larger current density and thereby steeper magnetic gradient. Density increase in the current sheet becomes larger in Cr-run, as electromagnetic forces do not accelerate plasma away as efficiently. Note that the inflow speed $v_{x}$ differs somewhat from the one prescribed as boundary condition: this is because the boundary condition sets the average velocity of particles injected at the boundary, but even the boundary cells may contain also particles that have arrived from elsewhere in the simulation. Therefore, the total flow velocity in the simulation is not necessarily equal to the boundary condition even at the boundary. As the inflow is subsonic, it is not completely dictated by the boundary condition anywhere in the simulation domain and may be, e.g., compressed and decelerated. In these runs, some deceleration occurs right at the inflow boundary, but not significantly from there to the current sheet boundary. In Lr-run, the flow actually accelerates a bit on the way in.

Figure 5 shows corresponding profiles within the current sheet, along the outflow direction, the last two panels now being reconnected magnetic field component $B_{x}$ and ion outflow speed $v_{z}$. Here, differences between the two runs are more striking. In Lr-run, there is a large dip in current density in the central region, as the current sheet is widened there. The reconnected flux density $\mathrm{Bx}$ increases rapidly with distance from the $\mathrm{x}$-point until the edge of the region of increased resistivity, behind which it slightly decreases. In Cr-run, $B_{x}$ increases very slowly along the entire outflow until the box boundary. Plasma acceleration, as shown by the slope of the $\mathrm{v}_{\mathrm{z}}$ curve, is largest in the central region in Lr-run and near the box edges in Cr-run. This is compatible with the behavior of $\mathrm{B}_{\mathrm{x}}$, which is very small in Cr-run and to which the accelerating $\vec{J} \times \vec{B}$ force is proportional. Note also that in Lr-run density and temperature, and thus also thermal pressure, increase somewhat with distance from the x-point. The

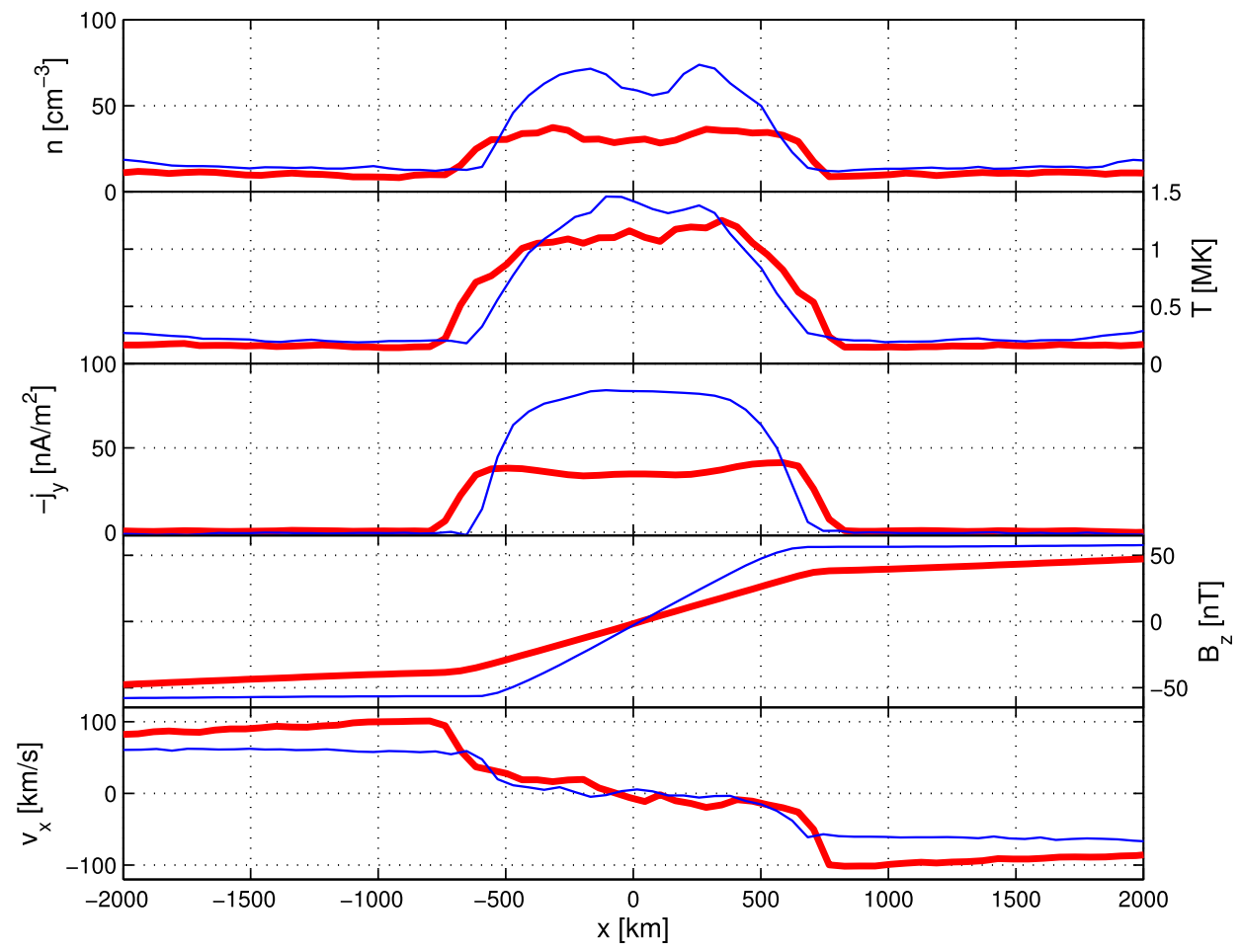

FIG. 4. Ion density, temperature, current density, reconnecting magnetic flux density $\mathrm{B}_{\mathrm{z}}$ and inflow speed $\mathrm{v}_{\mathrm{x}}$ on the central normal of the current sheet, i.e., the $\mathrm{x}$ axis, at $90 \mathrm{~s}\left(450 \omega_{\mathrm{ci}}{ }^{-1}\right)$ after simulation start. Thin blue curves describe Cr-run and thick red curves Lr-run. 


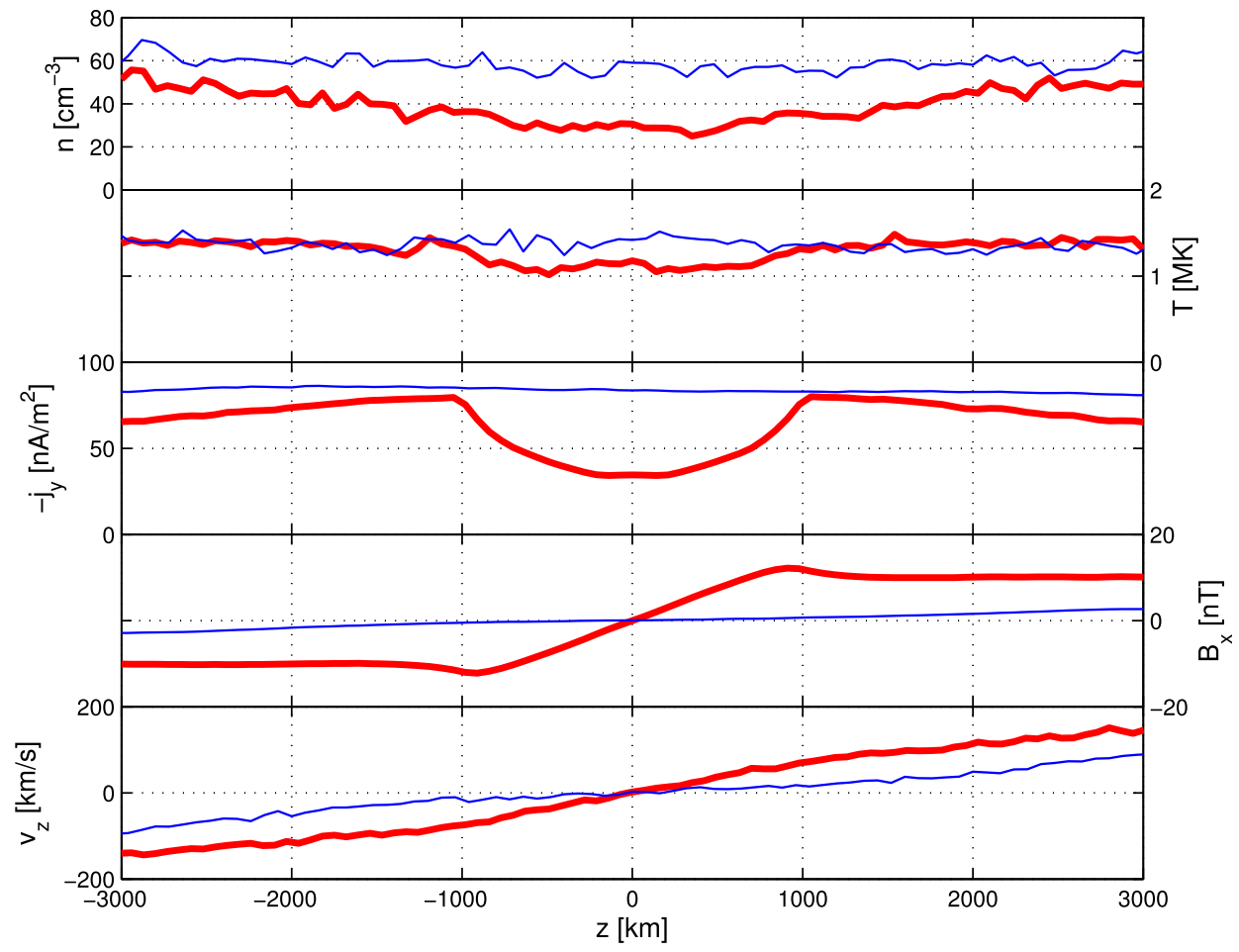

FIG. 5. Ion density, temperature, current density, reconnected magnetic flux density $B_{x}$ and outflow speed $\mathrm{v}_{\mathrm{z}}$ on the $\mathrm{z}$ axis, i.e., in the centre of the current sheet along the outflow direction, at $90 \mathrm{~s}\left(450 \omega_{\mathrm{ci}}{ }^{-1}\right)$ after simulation start. Thin blue curves describe Cr-run and thick red curves Lr-run.

$\vec{J} \times \vec{B}$ force therefore does work against pressure gradient, in addition to accelerating the ions. This is not the case in $\mathrm{Cr}$ run, where density and temperature are constant along the current sheet.

\section{Time development}

The inflow and outflow speeds of ions are shown as a function of time in Figure 6. The thick red curves are again for Lr-run and thin blue for Cr-run. The inflow speed (dashed line) is measured near but outside the diffusion region and the outflow speed (solid line) near the box boundary (places marked in Fig. 1). After simulation start, a high peak is seen in the inflow velocity at $10-25 \mathrm{~s}$ when plasma flows fill the almost empty simulation box and the decompression front

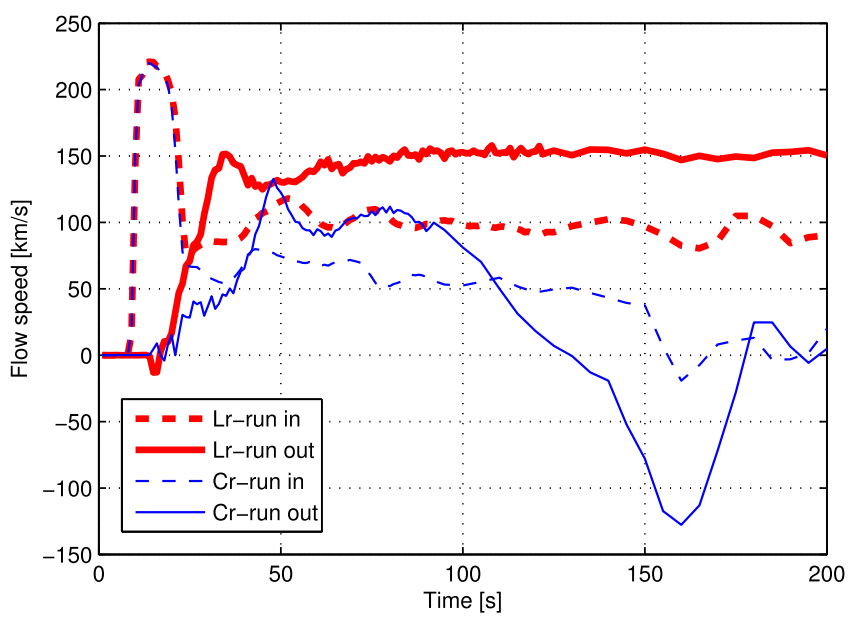

FIG. 6. Inflow speed to and outflow speed from the reconnection region. Thin blue curves describe Cr-run and thick red curves Lr-run. Dashed line: inflow speed, solid line: outflow speed. Measurement points are $\mathrm{x}=-1000 \mathrm{~km}, \mathrm{y}=\mathrm{z}=0$ for inflow and $\mathrm{z}=3000 \mathrm{~km}, \mathrm{x}=\mathrm{y}=0$ for outflow, marked in Fig. 1. passes the measuring point. After that differences between the two runs start to arise.

The inflow speed, as measured at $\mathrm{x}=-1000 \mathrm{~km}$, $\mathrm{y}=\mathrm{z}=0$ (marked in Fig. 1), settles at $100 \mathrm{~km} / \mathrm{s}\left(0.29 \mathrm{v}_{\mathrm{A} 0}\right)$ in Lr-run, well in line with the inflow boundary condition, and at a somewhat lower value in Cr-run. Due to less efficient reconnection, a larger pressure builds up in the simulation domain in Cr-run, and that opposes and decelerates the inflow right at the inflow boundary. The reconnection rate $v_{i n} / v_{A}$ in is approximately 0.35 in Lr-run, with similar temporal fluctuations as in the $v_{i n}$ curve in Fig. 6. In Cr-run, the reconnection rate is about 0.2 during the steadiest phase (between 60 and $110 \mathrm{~s}$ ) before reconnection in that run ceases.

In Lr-run, the outflow speed rises rapidly between 20 and $35 \mathrm{~s}$ of simulation time, then after minor oscillation stabilizes at $150 \mathrm{~km} / \mathrm{s}\left(0.43 \mathrm{v}_{\mathrm{A} 0}\right)$. In Cr-run, the outflow speed takes more time to rise to its peak value. This may be connected to the larger plasma pressure in the central current sheet: it takes time to build up that pressure. As $\left|v_{z}\right|$ increases all the way from the x-point to the boundaries (Fig. 5), the outflow jets probably have not reached their full speed within the simulation domain.

In Cr-run, the outflow speed stays semi-stable for about $50 \mathrm{~s}\left(250 \mathrm{\omega}_{\mathrm{ci}}{ }^{-1}\right)$. Then, it starts to decrease and eventually becomes negative. This happens because reconnection ceases and the x-point at the center of the box transforms into "an o-point" (not shown). Magnetic field lines merge at the boundaries of the box, exhibiting a kind of numerical boundary effect reconnection. The behavior of the Cr-run from $100 \mathrm{~s}$ onward is thus not physical. The resulting plasmoid, which is initially elongated along the current sheet, then contracts to assume a nearly circular shape. This contraction manifests itself as the negative excursion in the "outflow" speed. In Lr-run, reconnection process and outflow speed stay stable. Here, it is worth noting that at the 
boundary condition inflow speed of $100 \mathrm{~km} / \mathrm{s}$ it takes $30 \mathrm{~s}$ for the plasma to travel from the boundary to the center of the box. The outflows thus attain full speed in Lr-run quite rapidly, in flow-through timescale.

\section{Energy}

Figure 7 shows the time development of different energy fluxes in the simulations. In the first panel is the kinetic energy carried by protons. As the boundary conditions in the runs are the same, also the kinetic energy injection rate is the same (black dashed line). Kinetic energy of protons exiting the simulation box is shown by the solid lines.

In Lr-run, the kinetic energy exit rate is, after stabilization, about five times the kinetic energy input rate. This shows that energy is transferred from the electromagnetic field to the particles in the simulation. Note, however, that the kinetic energy exit rate takes $70 \mathrm{~s}$ to reach its stable level, which is twice as long as it takes for the outflow speed to reach its maximum level. The density grows in both the inand out-flow regions still after the outflow jets have reached their steady-state speed.

Another way of looking at this is the lower panel in Fig. 7, which shows the net Poynting flux into the current sheet region $(|\mathrm{x}|<1000 \mathrm{~km},|\mathrm{z}|<2500 \mathrm{~km})$. In Lr-run, it stays at a large positive value; and as the magnetic configuration is time-independent, this means that magnetic energy is being converted into other forms, as is expected in reconnection. In Cr-run, the Poynting flux into the reconnection region is most of the time greater than in Lr-run. However, the configuration in Cr-run is not time-independent, wherefore this flux does not represent energy conversion rate. It reflects the growth of magnetic energy stored in the central part of the simulation box in Cr-run.

The rate of Poynting flux destruction is much greater than the kinetic energy gained by protons even in Lr-run. The difference is approximately consumed by Joule heating, which can be calculated in the simulation as $P_{\mathrm{J}}=\eta \cdot J^{2}$, where $J$ is the total current density. This is illustrated in Fig. 8, which shows the energy budget of Lr-run. Ingoing energy fluxes are represented by negative values as areas below the zero line, while outgoing fluxes are positive. For technical reason, different fluxes are calculated at slightly different locations: The Poynting fluxes are calculated at surfaces within the simulation domain and Joule heating as a volume integral bounded by the Poynting flux calculation surfaces, while kinetic energy fluxes are calculated at the edge of the simulation domain. Therefore, the kinetic energy fluxes are only approximately comparable with other numbers. Note also that kinetic energy here is the sum of kinetic energies of individual protons: it includes both bulk flow and thermal energy.

Ingoing kinetic energy is, directly from the boundary condition, constant in time. Poynting flux dominates the ingoing energy flux. The order is the opposite in outgoing fluxes, kinetic being larger. But even the total outgoing energy flux is smallish compared to the ingoing flux. The budget becomes approximately balanced only when Joule heating, represented by the brownish area, is added to the outgoing fluxes.

\section{E. Other runs}

In addition to the two runs described above, a number of test runs were performed to see if the solution depends
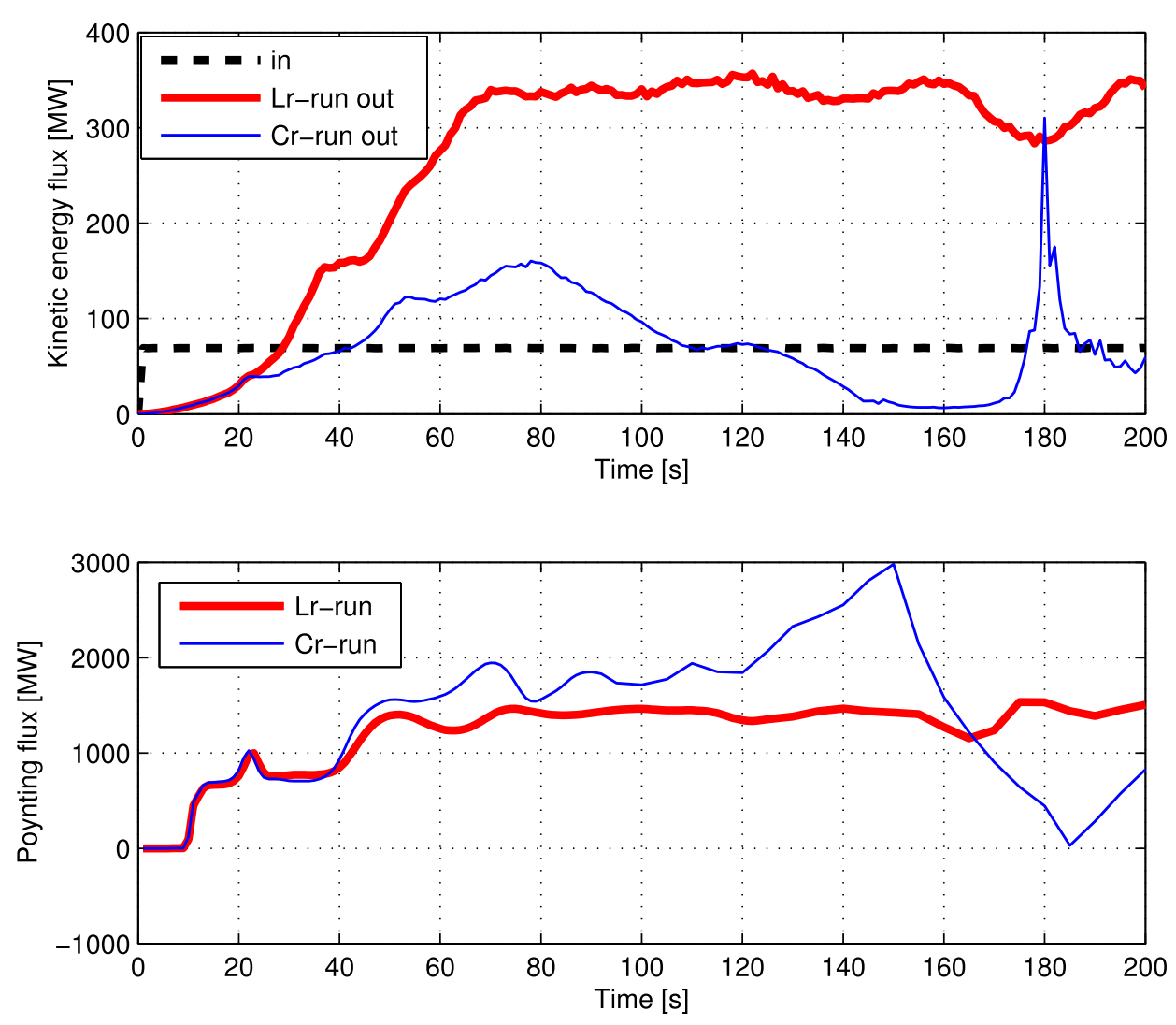

FIG. 7. First panel: kinetic energy exit rate from the simulation box with particles reaching the simulation boundary. Thick red curve for Lr-run and thin blue for Cr-run, black dashed line: kinetic energy injected into the simulation box (same for both runs). Second panel: Net Poynting flux into the reconnection region. Note that these numbers are not precisely comparable to the kinetic energies, as they are calculated in a different location. 


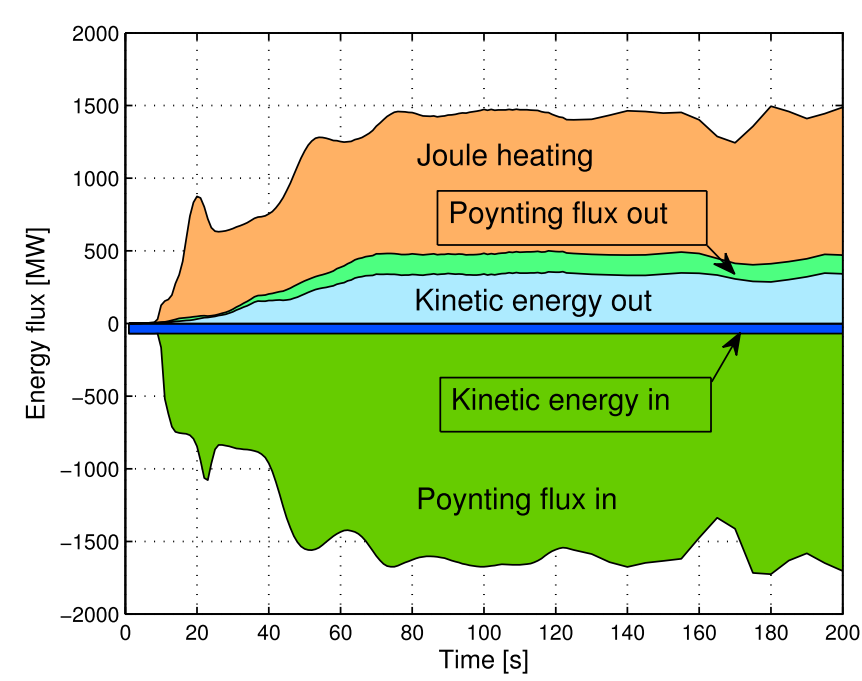

FIG. 8. Energy budget of Lr-run as an area stack plot. Green and blue areas below the zero-line represent magnetic and kinetic energy fluxes into the reconnection region. Light blue and light green areas above the zero-line represent corresponding fluxes out of the reconnection region. Orangebrown area represents the resistive dissipation $\eta j^{2}$ ("Joule heating") in the reconnection region.

crucially on some simulation parameter. We will not analyze these control runs in detail, but we outline some main results here.

The control runs were made with doubled grid cell size $(100 \mathrm{~km})$. If no other parameters are changed, the lower resolution solution is similar to the better resolution one and has only slight differences in numerical values. However, one minor difference during the initialization turns out to have a potentially decisive effect on reconnection ignition and stability. The tendency of the magnetic field to advance a bit faster near the boundary is smaller when resolution is coarser. This is probably the reason why reconnection stays stable even without localized resistivity, i.e., in coarserresistivity equivalent of the Cr-run, for $300 \mathrm{~s}$ (the entire duration of the run, not shown).

The simulations examined in this paper are threedimensional but represent an essentially two-dimensional configuration. To check that the box thickness in y direction does not affect the results, we doubled it from 1000 to $2000 \mathrm{~km}$ (from 10 to 20 cells with $100 \mathrm{~km}$ resolution). Reconnection attained full outflow speed a few seconds earlier but then stabilized at exactly the same speed and same configuration. No significant 3D structure was observed in any of the runs.

We also tested omitting the Hall term from the electric field equation. The effect was decisive: without the Hall term only slow diffusion occurred. Maximum outflow speed was a little lower than the inflow speed. A time-independent state was not reached, but magnetic field kept piling up. Thus, in the analyzed hybrid model runs the Hall term is essential for producing reconnection.

\section{DISCUSSION AND CONCLUSIONS}

Using localized resistivity is a common way in reconnection simulations to make an $\mathrm{x}$-line form at the desired place in MHD and hybrid simulations. It is typically used when the initial condition does not include an explicit perturbation of the magnetic field, a "seed x-line" that was the starting point for reconnection in, e.g., the GEM challenge ${ }^{7}$ and in fully kinetic models where electric field is calculated self-consistently without a resistivity parameter. A variation of the localized resistivity is to use time-varying resistivity, which is dependent on simulated quantities, such as the current density. That kind of an arrangement has been used to simulate "spontaneously" time-varying reconnection. ${ }^{24,25}$

In this paper, we used another method to break the translational symmetry of the current sheet, namely the gradient in inflow speed. ${ }^{14,28}$ It suffices to bend the inflowing magnetic field lines so that an $\mathrm{x}$-line forms at the desired place. However, as shown by the comparison of Cr- and Lr-run, localized resistivity can have a major effect even if it is not necessary to initiate reconnection. This wakes up the question, how well can one generalize results obtained with localized resistivity? On the other hand, even though localized resistivity is considered an artificial construction in reconnection simulations including the one presented in this paper, there are arguments for anomalous resistivity ${ }^{26,27}$ that could have a similar effect in real plasmas. Global simulations of different space plasma environments most often have only constant resistivity, but adding a current-density term has been shown to allow reproducing, e.g., the magnetospheric substorm cycle. ${ }^{24}$

A current-dependent anomalous resistivity has been used also, e.g., by Sato and Hayashi in driven reconnection in box MHD simulations: ${ }^{28}$ in their simulations, it causes a resistivity pattern, which is spatially quite similar to the one used in our Lr-run, but not temporally. Current-dependent resistivity starts to have effect only when current sheet has grown thin and intense, which means that there is a magnetic flux buildup phase followed by a fast energy release when the current density threshold for anomalous resistivity is exceeded. In our simulations, such stepwise behavior is not observed, as resistivity is temporally constant. Sato and Hayashi find that after the onset the later phases of reconnection are controlled by boundary conditions, not resistivity scheme. This is consistent with the first $100 \mathrm{~s}\left(500 \mathrm{\omega}_{\mathrm{ci}}{ }^{-1}\right)$ of our simulations, where $\mathrm{Cr}$-run produces similar if somewhat weaker reconnection than Lr-run. However, when continuing the runs longer, the same boundary conditions fail to keep the reconnection process going unless assisted by localized resistivity.

We showed that an important part of magnetic energy in our reconnection simulations goes to Joule heating. As resistivity in the code is an imposed parameter controlling magnetic diffusion, and does not arise from physical modelling of current-carrier interactions, the energy taken from the magnetic field by resistive diffusion is not transferred to the particles. Here, it is worth noting that a different electric field is used in calculating the Lorentz force and the Faraday's law:

$$
m_{i} \frac{d \overrightarrow{v_{i}}}{d t}=q_{i}\left(\vec{E}+\overrightarrow{v_{i}} \times \vec{B}\right)=q_{i}\left(-\overrightarrow{U_{e}} \times \vec{B}+\overrightarrow{v_{i}} \times \vec{B}\right),
$$

i.e., the electric field in the Lorentz force is the ideal advection electric field of the electron fluid without resistivity contribution, whereas 


$$
\begin{aligned}
\frac{\partial \vec{B}}{\partial t} & =-\nabla \times \vec{E}=-\nabla \times\left(-\vec{U}_{e} \times \vec{B}+\eta \vec{J}\right) \\
& =\nabla \times\left(\vec{U}_{e} \times \vec{B}\right)-\nabla \times \eta \vec{J},
\end{aligned}
$$

i.e., the Faraday's law includes a diffusion term proportional to resistivity. Dissipation of electromagnetic energy can be calculated as

$$
\begin{aligned}
\vec{E} \cdot \vec{J} & =\left(-\vec{U}_{e} \times \vec{B}+\eta \vec{J}\right) \cdot \vec{J} \\
& =(\vec{J} \times \vec{B}) \cdot \vec{U}_{e}+\eta J^{2} .
\end{aligned}
$$

In the model, the first term of the above formula corresponds to the electric field affecting ions through the Lorentz force, while the second term $\eta J^{2}$ represents the resistive heating, called Joule heating in Fig. 8. As this part of the electric field does not appear in the Lorentz force equation, it does not affect the ions. Nor is the energy transferred to electrons, as the electron temperature in the model is constant. Therefore, energy is not conserved in the model when resistive diffusion plays an important role. This naturally raises the question, should one try to include the lost energy back into the simulation to make the results more physical? However, it is not obvious how one should do this. Should it go to heating of the electron fluid, to extra heating of ions or maybe both to electrons and ions? We speculate that Joule heating energy loss may be one reason why the outflow velocity in our simulations remains clearly below the Alfvén speed, although one must remember that the theoretical expectation of outflow velocity being equal to Alfvén speed is only an orderof-magnitude estimate, not an exact prediction.

Our simulations show translational symmetry in the y direction, broken only by small random fluctuations. In an earlier 3D hybrid simulation by Nakamura et al., complex 3D dynamical effects were observed at the front of the reconnection jet. ${ }^{29}$ Because our focus was on obtaining a long-lasting steady reconnection, to keep computing time reasonable, we used a thin simulation domain that does not have enough space for any complex structure to develop in the y-direction. Furthermore, our background resistivity, i.e., magnetic diffusivity, was considerably larger than in Nakamura et al.'s work to ensure long-time numerical stability, which also inhibits the growth of small-scale structures.

To summarize, we have illustrated that a hybrid simulation code can produce continuous reconnection in a box with open boundary conditions and forced inflow. Reconnection ignites in the same timescale that it takes for the plasma to flow through the simulation box at the forced inflow speed, despite the fact that some of the magnetic energy is also lost in diffusion due to the non-conservative nature of the simulation. The efficiency and stability of reconnection are crucially enhanced when an artificial localized resistivity increase is applied in the region containing the $\mathrm{x}$-line. With localized resistivity, the kinetic energy of plasma increases about fivefold while flowing through the simulation domain.

\section{ACKNOWLEDGMENTS}

The work of T. L. and R. J. was supported by the Academy of Finland. The authors thank EU FP7 project IMPEx (Integrated Medium for Planetary Exploration, Project number 262863) for financial support. Figures 2 and 3 were created using the VisIt open source visualization tool.

${ }^{1}$ R. J. Walker, T. Ogino, J. Raeder, and M. Ashour-Abdalla, J. Geophys. Res. 98(A10), 17235, doi:10.1029/93JA01321 (1993).

${ }^{2}$ T. V. Laitinen, T. I. Pulkkinen, M. Palmroth, P. Janhunen, and H. E. J. Koskinen, Ann. Geophys. 23, 3753 (2005).

${ }^{3}$ T. V. Laitinen, M. Palmroth, T. I. Pulkkinen, P. Janhunen, and H. E. J. Koskinen, J. Geophys. Res. 112, A11201, doi:10.1029/2007JA012352 (2007).

${ }^{4}$ J. E. Ouellette, B. N. Rogers, M. Wiltberger, and J. G. Lyon, J. Geophys. Res. 115, A08222, doi:10.1029/2009JA014886 (2010).

${ }^{5}$ B. Tan, Y. Lin, J. D. Perez, and X. Y. Wang, J. Geophys. Res. 116, A02206, doi:10.1029/2010JA015580 (2011).

${ }^{6}$ R.-F. Lottermoser, M. Scholer, and A. P. Matthews, J. Geophys. Res. 103(A3), 4547, doi:10.1029/97JA01872 (1998).

${ }^{7}$ J. Birn, R. Nakamura, E. V. Panov, and M. Hesse, J. Geophys. Res. 116, A01210, doi:10.1029/2010JA016083 (2011).

${ }^{8}$ J. Birn, J. F. Drake, M. A. Shay, B. N. Rogers, R. E. Denton, M. Hesse, M. Kuznetsova, Z. W. Ma, A. Bhattacharjee, A. Otto, and P. L. Pritchett, J. Geophys. Res. 106(A3), 3715, doi:10.1029/1999JA900449 (2001); and references therein.

${ }^{9}$ M. Scholer, J. Geophys. Res. 94, 8805, doi:10.1029/JA094iA07p08805 (1989).

${ }^{10}$ J. Huba and L. Rudakov, Phys. Rev. Lett. 93, 175003 (2004).

${ }^{11}$ D. Krauss-Varban and N. Omidi, Geophys. Res. Lett. 22, 3271, doi:10.1029/95GL03414 (1995).

${ }^{12}$ W. Daughton, J. Scudder, and H. Karimabadi, Phys. Plasmas 13, 072101 (2006).

${ }^{13}$ W. Pei, R. Horiuchi, and T. Sato, Phys. Rev. Lett. 87, 235003 (2001).

${ }^{14}$ R. Horiuchi and T. Sato, Phys. Plasmas 1, 3587 (1994).

${ }^{15}$ D. Krauss-Varban, H. Karimabadi, and N. Omidi, Geophys. Res. Lett. 26, 1235, doi:10.1029/1999GL900211 (1999).

${ }^{16}$ J. Birn, K. Galsgaard, M. Hesse, M. Hoshino, J. Huba, G. Lapenta, P. L. Pritchett, K. Schindler, L. Yin, J. Büchner, T. Neukirch, and E. R. Priest, Geophys. Res. Lett. 32, L06105, doi:10.1029/2004GL022058 (2005).

${ }^{17}$ E. Kallio and P. Janhunen, J. Geophys. Res. 106, 5617, doi:10.1029/ 2000JA000239(2001); 107, SIA 1-1, doi:10.1029/2001JA000090 (2002).

${ }^{18}$ R. Jarvinen, E. Kallio, I. Sillanpää, and P. Janhunen, Adv. Space Res. 41, 1361 (2008).

${ }^{19}$ E. Kallio and P. Janhunen, Ann. Geophys. 21, 2133 (2003).

${ }^{20}$ E. Kallio, Geophys. Res. Lett. 32, 6107, doi:10.1029/2004GL021989 (2005).

${ }^{21}$ E. Kallio, S. Barabash, P. Janhunen, and R. Jarvinen, Planet. Space Sci. 56, 823 (2008).

${ }^{22}$ R. Jarvinen, E. Kallio, P. Janhunen, S. Barabash, T. L. Zhang, V. Pohjola, and I. Sillanpää, Ann. Geophys. 27, 4333 (2009).

${ }^{23}$ I. Sillanpää, Ph.D. Thesis, University of Helsinki, Helsinki, 2008.

${ }^{24}$ J. Schumacher, B. Kliem, and N. Seehafer, Phys. Plasmas 7, 108 (2000).

${ }^{25}$ J. Raeder, R. L. McPherron, L. A. Frank, S. Kokubun, G. Lu, T. Mukai, W. R. Paterson, J. B. Sigwarth, H. J. Singer, and J. A. Slavin, J. Geophys. Res. 106(A1), 381, doi:10.1029/2000JA000605 (2001).

${ }^{26} \mathrm{G}$. Wu, G. Huang, and H. Ji, Astrophys. J. 720, 771 (2010).

${ }^{27}$ L. P. Dyrud and M. M. Oppenheim, J. Geophys. Res. 111, A01302, doi:10.1029/2004JA010482 (2006).

${ }^{28}$ T. Sato and T. Hayashi, Phys. Fluids 22, 1189 (1979).

${ }^{29}$ M. S. Nakamura, H. Matsuto, and M. Fujimoto, Geophys. Res. Lett. 29, 88, doi:10.1029/2001GL013780 (2002). 